\title{
REGRESSÃO NÃO-LINEAR DE BLEASDALE: REVISITANDO OS PRESSUPOSTOS TEÓRICOS DE JAKOB NIELSEN
}

\author{
Marcos dos Santos (marcosdossantos_doutorado_uff@yahoo.com.br) - CASNAV - UFF \\ Thiago Leite Porto e Silva (thi.l.porto@ hotmail.com) - SENAI CETIQT \\ Rubens Aguiar Walker (rubens.walker@gmail.com) - UFF \\ Marcone Freitas dos Reis (marconefreis11@gmail.com) - UFF - SENAI CETIQT \\ Jéssica Alves de Souza (jessica.asouza@yahoo.com.br) - SENAI CETIQT
}

\begin{abstract}
RESUMO
Essa pesquisa tem o propósito de verificar se a satisfação dos usuários de smartphones possui algum tipo de correlação com o tempo para realização de uma atividade qualquer. Para isso, foi realizada uma pesquisa presencial com 30 usuários. Foi medido o tempo para a realização de uma atividade até a conclusão ou desistência da mesma. Cada respondente informava a sua satisfação ao realizar tal atividade de acordo com a Escala Likert de 5 pontos. Inicialmente, obteve-se o valor de -0,88 para o Coeficiente de Correlação Linear de Pearson, indicando uma correlação linear negativa forte entre a satisfação e o tempo de realização da atividade. Contudo, levando em consideração a simplicidade $e$ as limitações da Regressão Linear, foram pesquisadas outras regressões, a fim de permitir interpolações mais precisas. Pode-se testar uma série de regressões não-lineares, chegando-se à Curva de Bleasdale como a equação de melhor aderência aos dados coletados. A metodologia ora apresentada pode representar uma vantagem estratégica para os fabricantes de smartphones, uma vez que os desenvolvedores terão acesso ao tempo de operação que pode maximizar a satisfação do usuário para cada funcionalidade de um smartphone. A contribuição acadêmica desse trabalho repousa sobre o estabelecimento de uma métrica e de um modelo matemático desenvolvidos a partir dos pressupostos teóricos sobre usabilidade de Jakob Nielsen.
\end{abstract}

Palavras-chave: Escala Likert; Smartphone; Regressão Linear; Regressão Não-Linear; Modelo de Bleasdale.

Área: Gestão de projetos de desenvolvimento de produtos e serviços.

\section{INTRODUÇÃO}

Em um mundo em que a informação tem grande importância e trafega globalmente de uma forma extremamente rápida, cada vez mais são demandadas ferramentas que facilitem essa comunicação. Desta forma, a tecnologia da informação teve sua parcela de contribuição para os processos e serviços empresariais, além das estratégias competitivas de empresas e pessoas. (TORRES, 2015)

Com o tempo, não só as empresas viram a necessidade de uma comunicação dinâmica e globalizada, mas pessoas físicas começaram a utilizar o seu computador pessoal com enfoque de busca, compras, uso de mídia social entre outras. Isto acabou popularizando o uso e aumentando o número de pessoas com acesso à internet. (IBGE, 2015)

O volume de vendas de aparelhos smartphones é muito grande no mundo. Em 2015, foram enviados para as lojas 1.5 bilhões de celulares. Esse total é 11,9\% maior que no ano anterior (IDC, 2015). O IDC Brasil afirma que no mercado brasileiro em 2014 foram vendidos 104 
smartphones por minuto, tornando assim o Brasil o quarto país com maior número de vendas no mundo, atrás somente dos Estados Unidos, Índia e China.

Segundo Nielsen (2007) no século 19, a navegação pela internet era bem diferente de atualmente, pois hoje ela faz parte da rotina das pessoas, sendo considerada agora uma ferramenta. Nielsen identificou que se este instrumento for de fácil entendimento será de fato utilizado, caso contrário não. Krug (2014) acredita que os usuários não querem pensar no momento da utilização dos softwares. Qualquer aplicativo ou site que tenham funções que estejam a mais de dois cliques de distância ou, não seja de fácil entendimento, passa a ser considerado como um problema. Fato que ele considera como a primeira lei da usabilidade.

\section{FORMATAÇÃO GERAL}

O mercado de sistema operacional é restrito a 3 grandes marcas, cabendo a elas disputarem por quase todo o mercado. O Android, criado pela Google em 2008 é utilizado pela maioria das fabricantes atualmente. O iOS, criado pela Apple em 2007, é utilizado de forma exclusiva pela mesma. O Windows Phone, criado pela Microsoft em 2010, é utilizado por poucas empresas além da própria Microsoft. (SMART MUNDO, 2013)

Todos os competidores possuem aparelhos que, em sua maioria, rivalizam na mesma faixa de preço e possuem majoritariamente os mesmos hardwares construídos por parceiros. Até mesmo o chipset da parceira fabricante Qualcomm é igual. Ao verificar os modelos "top de linha" atual das 5 empresas que possuem maior participação de Market Share no Brasil, consegue-se identificar que 3 delas utilizam o mesmo parceiro de chipset, 4 delas utilizam Android e 3 utilizam o Chrome como navegador.

Tendo em vista a quantidade de software e hardware iguais no mercado de smartphones, o diferencial torna-se a usabilidade na interface do sistema.

Segundo o IBGE (PNAD, 2014) o telefone celular é usado para navegar na rede em 80,4\% das casas que possuem acesso á internet, diferentemente do computador que é usado em somente 76,6\% apresentando uma queda em comparação com o ano anterior. Com o aumento do uso de navegadores de internet pelo celular, algumas empresas podem estar perdendo uma vantagem competitiva utilizando o Chrome, que é padrão em várias marcas.

Com o intuito de fomentar a criação de navegadores próprios de cada marca, assim como a Apple possui o Safari, foi realizado um teste de usabilidade para identificar a influência que existe entre o tempo para realizar uma ação no navegador de internet e a satisfação do usuário. A partir dessa informação, os desenvolvedores poderão testar os seus novos navegadores de forma mais fácil e direcionada para o usuário. Assim, esta pesquisa tem o propósito de verificar de que maneira a duração para realizar uma ação no navegador de internet de um smartphone impacta na satisfação do usuário.

\section{METODOLOGIA}

Nielsen (1993) subdivide a usabilidade em eficiência, eficácia e satisfação. Nessa pesquisa, a eficiência foi medida como o tempo que um indivíduo leva para realizar uma ação indicada. A satisfação foi medida seguindo a escala Likert de 5 pontos, sendo eles: completamente satisfeito, parcialmente satisfeito, nem satisfeito e nem insatisfeito, parcialmente satisfeito e completamente insatisfeito. A eficácia foi medida com a conclusão ou não da tarefa.

Segundo Vergara (2003) as Pesquisas aplicadas são exploratórias, ou seja, tem como objetivo a maior finalidade com o problema, além de serem também descritivas, por estabelecer 
relação entre variáveis. A primeira pesquisa foi feita de forma quantitativa e foi realizada no mês de Outubro de 2016 utilizando a ferramenta SurveyMonkey, tendo sido recebidas 86 respostas, contendo em seu escopo 2 perguntas para identificação da amostragem e 1 para identificação das funcionalidades mais usadas nos smartphones, nesta pergunta poderia ser escolhida mais de uma função. A identificação na amostragem quanto à idade e gênero foi usada para observar a homogeneidade da amostra. Para verificar a representatividade da amostra foi utilizada uma comparação com o resultado obtido no PNAD 2014, pesquisa nacional por amostra de domicílios no Brasil, realizado pelo IBGE. A pesquisa foi exposta em diversos fóruns online sobre pesquisas colaborativas e, além disso, também foi respondida por conhecidos dos autores.

A pesquisa qualitativa e presencial, foi realizada no mês de março de 2017, tendo 30 participantes, sendo 15 homens e 15 mulheres, o método de busca dos participantes foi por proximidade, ou seja, são pessoas conhecidas dos autores. O teste foi realizado com 3 aparelhos, ou seja, 10 pessoas realizaram o teste com cada um. Os smartphones escolhidos foram: Motorola Moto X Segunda Geração, Galaxy S6 Edge e iPhone 6s Plus. A escolha destes modelos foi devido ao sistema operacional ser Android puro com o navegador Chrome, Android modificado com o navegador Internet e o iOS com o navegador Safari, respectivamente. Cada pessoa pesquisada utilizou somente aparelhos que não lhes eram familiares, a fim de não enviesar os resultados da pesquisa. Foi solicitado que os pesquisados completassem 9 ações em sequência relacionadas ao navegador do aparelho, quais sejam:

- Abrir a página do Facebook.com;

- Abrir uma nova aba;

- Abrir uma nova aba no modo Oculto/Privado;

- Fechar uma aba que foi aberta;

- Colocar o site Facebook.com nos favoritos;

- Abrir uma página dos favoritos;

- Apagar o histórico de websites acessados;

- Compartilhar o site do Facebook.com por e-mail; e

- Mudar o Buscador padrão para Bing.com.

Verificou-se o sucesso ou fracasso em realizar as ações, o tempo para realizar cada ação e a satisfação do usuário para cada ação.

\section{FUNDAMENTAÇÃO TEÓRICA}

\subsection{Usabilidade}

Moran (1981) definiu, baseado no IHC, a interface do sistema com o usuário como sendo a parte de um sistema computacional com o qual qualquer pessoa pode entrar em contato, sendo ela física, perceptiva ou conceitual.

A usabilidade tem por objetivo agir nas interfaces com o objetivo de permitir uma interação fácil, agradável, com eficácia e eficiência. A usabilidade então é, de fato, uma área dentro da Interação Humano-Computador. (NIELSEN, 1993)

Outra norma que também aborda questões relacionadas à usabilidade é a ISO 9126-1, criada em 2002 como uma parte da qualidade do produto na engenharia de software. Nesta área, a 
usabilidade foi abordada como a capacidade do produto software de ser compreendido, aprendido, operado e atraente ao usuário. Por conseguinte, para ser verificada e medida, a usabilidade precisa ser testada com usuários.

Kurg em 2014 utilizou-se do trabalho de Jakob Nielsen para gerar a visão dele de como a usabilidade deve ser melhorada. Assim, lançou as suas três leis:

- Evite que o usuário pense enquanto navega no software.

- A quantidade de cliques que o usuário fará não é importante, contanto que seja impensada e não ambígua.

- As quantidades de palavras no site devem ser mínimas.

Nielsen (1993) afirma baseado em diversos estudos que nem sempre os usuários sabem o que é bom para eles. Além disso, os usuários também possuem opiniões divergentes quando perguntadas sobre uma interface. Esses estudos confirmam a frase "Consumidores não sabem o que querem" dita por Steve Jobs (FORBES, 2011). Perguntar para o usuário o que ele deseja, antes de ter a ferramenta no mínimo parcialmente pronta, não resolverá qualquer problema na usabilidade.

O Trabalho do designer deve ser testado com usuários, imprescindivelmente. Vale ressaltar que os próprios designers não podem testar seus trabalhos porque, nesta visão, eles não são considerados usuários, assim como CEO (da própria empresa) e nenhum outro cargo, ou seja, o teste com usuários é determinante para o sucesso. (NIELSEN, 1993)

Para realizar um teste de usabilidade, os pesquisados tendem a pensar que é limitativo que o protótipo funcione totalmente, ou seja, cumpra o que foi prometido. Porém, é possível detectar sérios problemas de usabilidade mesmo no início, trabalhando com pequenos módulos de produto. Problemas resolvidos mais cedo costumam impactar menos nos projetos.

Para Krug (2010) o teste de usabilidade deve ser feito nas seguintes etapas:

Figura 1. Etapas de teste de Krug. Fonte: Krug (2010)

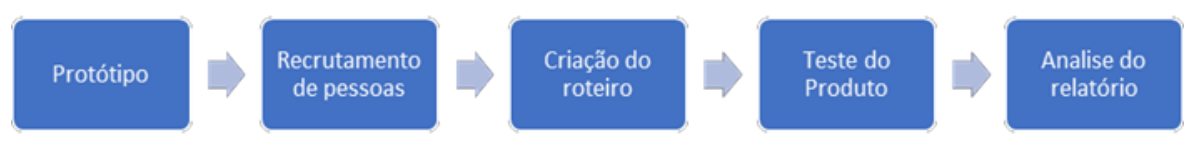

No teste de usabilidade devem ser analisados se o sistema é de fácil utilização, o tempo requerido para completar a ação, se as telas podem ser fáceis de serem memorizadas, a quantidade de erros e a satisfação do usuário. (NIELSEN, 1993)

Para Barbosa (2013) é limitativo que o sistema seja fiável, ou seja, a fiabilidade de um componente é a probabilidade deste desempenhar de forma adequada a função para que foi concebido, nas condições previstas e nos intervalos de tempo que tal é exigido. Caso se estabeleça uma comparação dos estudos de Nielsen (1993) com Barbosa (2013), pode-se verificar que ambos acreditam que a eficiência, a eficácia e a satisfação são o foco primordial da usabilidade, sendo a eficácia referente à taxa de erros e a eficiência referente ao tempo requerido para completar uma ação. A variável eficiência é influenciada pela facilidade de utilização e a memorização da tela.

\subsection{Escala Likert}

Os fatos e fenômenos de interesse podem ser compreendidos pela mensuração, por isso, a mensuração é algo presente em quase todas as ciências. (SILVA, COSTA; 2014) 
A mensuração é definida como atribuição de símbolos, que normalmente são usados números, a alguma propriedade dos objetos que se deseja obter a medição. Sendo assim a medição é um processo de representação, relacionando o mundo real com este sistema simbólico. (MARLI, 1996; FINKELSTEIN, 2003, 2009)

As escalas no grande universo das ciências comportamentais, que não diferem tanto de outras ciências, são fincadas em pressupostos particulares e desenvolvidas por modelos específicos (COSTA, 2011).

Psicometria, segundo Erthal (1987), é uma área da psicologia que interage dois mundos completamente diferentes, ciências exatas e psicologia, ou seja, ela utiliza estatística para medir algo da psicologia. Existem diversas escalas que realizam as medidas psicométricas como a escala Stevens e a de Likert, que são as mais famosas.

Criado por Rensis Likert em 1932, a escala Likert é o modelo mais utilizado e debatido entre os pesquisadores e serve para mensurar atitudes no contexto das ciências comportamentais. (SILVA, COSTA; 2014)

Existem diversos tipos de escalas de medida, como escala nominal, escala ordinal, escala intervalar, escala de razão, entre outras. A escala Likert pertence ao grupo ordinal, ou seja, uma escala de grandeza nas categorias é a característica principal dessa escala. (ALEXANDRE et al, 2003)

Este método possibilita a medição do grau da intensidade tendo em vista o objeto de estudo. Tal intensidade vem na forma de uma afirmativa em que os respondentes possuem cinco opções de resposta: discordo totalmente, discordo parcialmente, não concordo e nem discordo, concordo parcialmente e concordo totalmente. (MAFRA, 1999)

O item intermediário "nem insatisfeito e nem satisfeito", considerado neutro, utilizado nas pesquisas de três pontos, ou seja, somente três opções de resposta, pode conduzir o entrevistado a ter uma tendência de marcar mais positivamente em sua resposta. Enquanto o de sete pontos pode confundir o respondente na hora da resposta. Sendo assim é importante analisar bem se esses modelos que são considerados similares a Likert. (ALEXANDRE et al, 2003)

\subsection{Coeficiente de correlação}

Correlação é um método estatístico utilizado para identificar uma possível associação linear entre duas variáveis. O termo correlação é usado para referenciar uma associação, conexão ou qualquer forma de relação e correspondência. (MUKAKA, 2012)

Segundo Moretin e Toloi (2009) pode ser identificado a correlação entre duas variáveis utilizando a correlação de Pearson:

$$
r=\frac{n\left(\sum X Y\right)-\left(\sum X\right)(Y)}{\sqrt{n\left(\sum X^{2}\right)-\left(\sum X\right)^{2}} \cdot \sqrt{n\left(\sum Y^{2}\right)-\left(\sum Y\right)^{2}}}
$$

\subsection{Regressão Matemática}

A ferramenta estatística utilizada para modelar e explorar as relações entre duas ou mais variáveis de maneira determinística é chamada de análise da regressão. Até hoje o cálculo da regressão é a ferramenta mais utilizada em diversos ramos. (MONTGOMERY, RUNGER, 2012) 
Existem diversos métodos estatísticos que ajudam a estudar a associação entre duas ou mais variáveis, porém, a que ocupa lugar de destaque é a teoria de regressão e correlação. (NAGHETTINI; PINTO, 2007)

Segundo Carvalho et al (2016) existem diversos tipos de regressões lineares e a mais básica é chamada de regressão linear simples, que consiste em definir a correlação linear entre as variáveis (independente e dependente).

A literatura de Doane e Seward (2014) aponta a fórmula para regressão linear simples como sendo:

Onde:

$$
Y=\beta_{0}+\beta_{1} X+e
$$

$\mathrm{y}=$ variável dependente

$\beta_{0}$ e $\beta_{1}=$ coeficientes desconhecidos da regressão; $\mathrm{x}=$ valor da variável independente;

$\mathrm{E}=$ erros ou resíduos da regressão.

Para estimar os valores de $\beta_{0}$ e $\beta_{1}$, utilizam-se as fórmulas de estimativa de mínimos quadrados a seguir.

$$
\begin{gathered}
\hat{\beta}_{0}=\bar{y}-\bar{x} \\
\hat{\beta}_{1}=\frac{\sum_{i=1}^{n} y_{i} x_{i}-\frac{\left(\sum_{i=1}^{n} y_{i}\right)\left(\sum_{i=1}^{n} x_{i}\right)}{n}}{\sum_{i=1}^{n} x_{i}^{2}-\frac{\left(\sum_{i=1}^{n} x_{i}\right)^{2}}{n}}
\end{gathered}
$$

Onde:

$$
\begin{gathered}
\bar{y}=\frac{1}{n} \sum_{i=1}^{n} y_{i} \\
\bar{x}=\frac{1}{n} \sum_{i=1}^{n} y_{i}
\end{gathered}
$$

Existem diversos modelos não lineares. Os modelos mais conhecidos, segundo Mattos (2013), são:

- Modelo de Mitscherlich:

$$
Y=\alpha\left(1-10^{-\gamma(X+\theta)}\right)+\varepsilon
$$

- Modelo Logístico:

$$
Y=\frac{\theta}{1+e^{-(\alpha+\beta)}}+\varepsilon
$$

- Modelo de Gompertz:

$$
Y=e^{-\alpha+\beta p X}+\varepsilon
$$


- Modelo de Michaelis-Mentel:

$$
Y=\frac{\theta_{1} X}{X+\theta_{2}}+\varepsilon
$$

\section{MODELAGEM MATEMÁTICA}

Para tratar os 270 dados obtidos foi utilizado o programa EXCEL 2010. Plotou-se o gráfico a seguir a partir de todas as medições de tempo e satisfação. Adensando esta análise, também foi calculada a média para cada satisfação.

Figura 2. Medições de satisfação x Tempo. Fonte: Autores (2017)

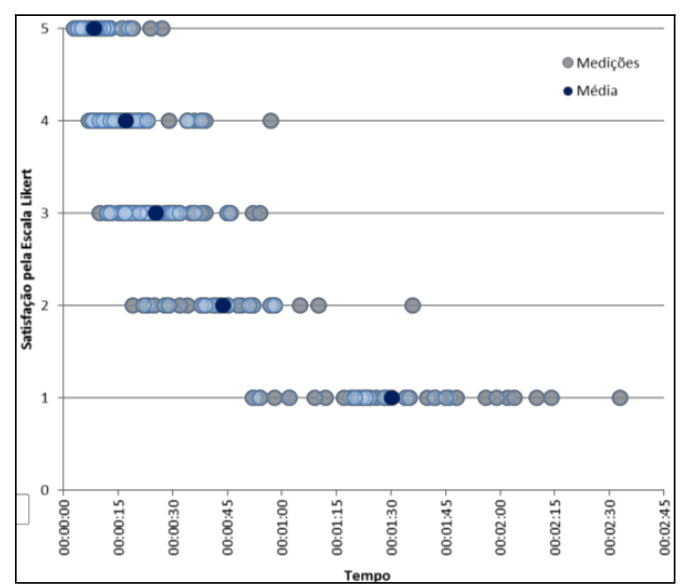

O gráfico possui duas variáveis, sendo no eixo das abcissas as medições do tempo e no eixo das coordenadas a satisfação na escala Likert em números. Pode-se identificar que, quanto maior o tempo que o usuário demora para realizar a ação, menos satisfeito ele fica. As médias de tempo para cada número de satisfação da escala Likert estão dispostas na tabela a seguir.

Tabela 1. Exemplo de tabela

\begin{tabular}{|c|c|}
\hline Satisfação & Tempo médio (mm:ss) \\
\hline $\mathbf{1}$ & $01: 30$ \\
\hline $\mathbf{2}$ & $00: 44$ \\
\hline $\mathbf{3}$ & $00: 25$ \\
\hline $\mathbf{4}$ & $00: 17$ \\
\hline $\mathbf{5}$ & $00: 08$ \\
\hline
\end{tabular}

É possível identificar que a queda na satisfação é acentuada com o aumento do tempo. Podese visualizar somente pela média, que tempos acima de 9 segundos já podem deixar os usuários parcialmente satisfeitos com a ação, enquanto com menos de um minuto o usuário já estará no limiar entre parcialmente insatisfeito e completamente insatisfeito.

Com objetivo de verificar a existência de uma correlação entre as variáveis de satisfação e tempo utilizou-se a fórmula da correlação de Pearson, no qual foi identificado que existe uma correlação negativa forte cujo $r=-0,88$. Sendo assim, de fato existe uma correlação, apontando que ela deva ser analisada com maior profundidade. 
De posse destes resultados, os desenvolvedores de aplicativos podem entender melhor a percepção dos usuários, sabendo quanto tempo no máximo os usuários estão dispostos a gastar em cada operação em um determinado smartphone de maneira que mantenham uma percepção positiva quanto à usabilidade do mesmo.

Dada a limitação da regressão linear, buscou-se um modelo de maior aderência aos dados. Assim, chegou-se ao modelo de Bleasdale, sendo esta a regressão não linear que mais se ajusta às variáveis satisfação $\mathrm{x}$ tempo. A figura 3 a seguir, mostra um ranking com as regressões que melhor se ajustaram aos dados, onde se percebe que a regressão linear ocupa a $20^{\mathrm{a}}$ posição.

Figura 3. Rank dos modelos ajustados. Fonte: Autores (2017)

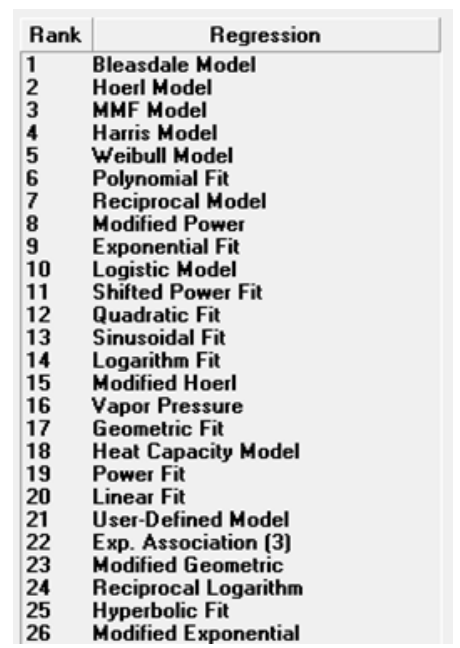

O modelo de Bleasdale é uma regressão exponencial que tende a zero no eixo das abcissas. Isso indica que a queda na satisfação com o tempo cai de forma abrupta.

Figura 4. Satisfação x tempo e o modelo de Bleasdale. Fonte: Autores (2017)

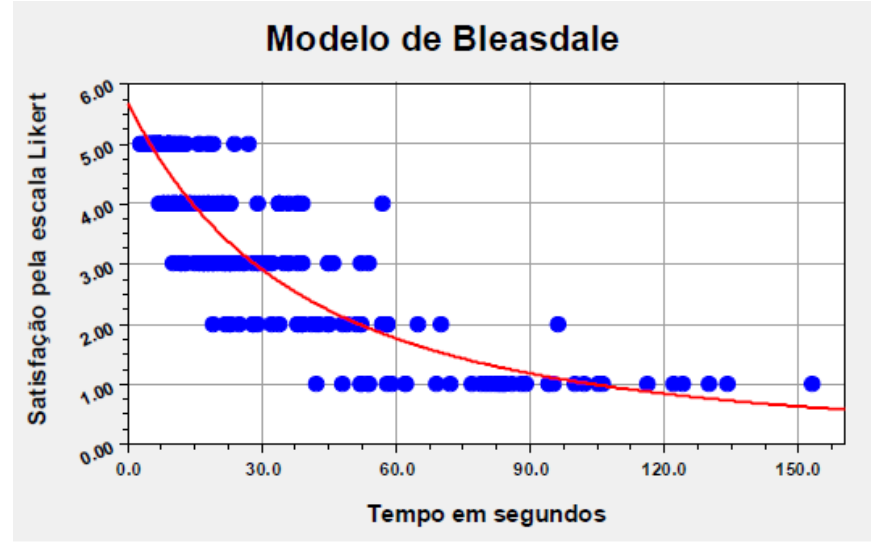

Sua curva é dada por:

$$
y=(a+b x)^{\frac{-1}{c}}
$$

Onde:

$\mathrm{y}=$ satisfação em escala Likert numérica

$\mathrm{x}=$ tempo em segundos

Os coeficientes da curva foram calculados, chegando-se aos seguintes resultados: 


$$
\begin{aligned}
& a=4.25462725229 e^{-1} \\
& b=5.54199247344 e^{-3} \\
& c=4.92789764985 e^{-1}
\end{aligned}
$$

Sendo assim, o desenvolvedor ao ter o tempo que foi demandado para realizar uma ação em seu navegador, pode lançar mão do modelo de Bleasdale, substituindo o x por esse valor de tempo, considerando os valores calculados para os parâmetros a, b e c indicados acima, a fim de se chegar à informação referente a satisfação em escala Likert numérica esperada.

\section{CONSIDERAÇÕES FINAIS}

Na pesquisa aqui apresentada, observou-se o binômio tempo de operação versus satisfação com relação ao navegador de internet. Em um primeiro momento, chegou a um Coeficiente de Correlação Linear de Pearson, $r=-0,88$ que por si só indica uma correlação linear negativa forte, permitindo a utilização da regressão linear para uma análise estatística do fenômeno.

Contudo, a partir de uma análise mais profunda, chegou-se à Regressão de Bleasdale, sendo esta a curva de melhor aderência aos dados. Com ela, a partir da satisfação que se deseja para uma determinada função de um smartphone, pode-se calcular o tempo máximo que o operador estará disposto a gastar com aquela operação.

Com esse estudo, espera-se trazer uma significativa contribuição para a sociedade uma vez que os smartphones já fazem parte do cotidiano de milhares de pessoas, seja no trabalho, no estudo ou no lazer. Assim, melhorar a usabilidade de hardwares e softwares dos referidos aparelhos, necessariamente perpassa pela melhoria da qualidade de vida das pessoas.

\section{REFERÊNCIAS}

ALEXANDRE, J. et al. Análise o número de categorias da escala likert aplicada à gestão pela qualidade total através da teoria da resposta do item. ENEGEP. Ouro Preto, Minas gerais, Brasil, 21 a 21 de out. 2003.

BARBOSA, F. Introdução á fiabilidade de sistemas elétricos de energia, Faculdade de Engenharia Universidade do Porto. 2013.

CARVALHO, T.C.; PEREIRA, K. R. A.; OLIVEIRA, M. A.; ANDRADE, A.R.O. Análise comparativa entre métodos de previsão de demanada - Um estudo aplicado a indústria de gás natural no Brasil. ENEGEP. João Pessoa - PB, Brasil, 03 a 06 de outubro, 2016.

COSTA, F. J. Mensuração e desenvolvimento de escalas: aplicações em administração. Rio de Janeiro: Ciência Moderna, 2011.

DOANE, D. P.; SEWARD, L. E. Estatística Aplicada à Administração e Economia. 4a .ed. São Paulo: Mc Graw Hill, 2014. 864p.

ERTHAL, C. Manual de psicometria. $8^{\circ}$ Edição. Jorge Zahar Ed., Rio de Janeiro, 1987. FORBES. Five Dangerous Lessons to Learn From Steve Jobs. Disponível em:

$<$ https://www.forbes.com/sites/chunkamui/2011/10/17/five-dangerous-lessons-to-learn-fromsteve-jobs/\#11f359b3a95c>. Acesso em: 01 de Abr de 2017.

FINKELSTEIN, L. Widely-defined measurement: An analysis of challenges. Measurement, 42, p.1270-1277, 2009. 
FINKELSTEIN, L. Widely, strongly and weakly defined measurement. Measurement, 34, p. 39-48,2003.

IBGE. Pesquisa Nacional por Amostra de Domicílios (PNAD) 2014. Disponível em: < http://biblioteca.ibge.gov.br/visualizacao/livros/liv94935.pdf>. Acesso em: 24 de Abril de 2017.

IDC. Worldwide Smartphone 2015-2019 Forecast and Analysis. Disponível em: < http://www.idc.com/getdoc.jsp?containerId=254912 >. Acesso em: 18 de Nov. de 2016.

IDC. Estudo da IDC Brasil aponta que, em 2014, brasileiros compraram cerca de 104 Smartphones por minuto. Disponível em:

<http://br.idclatin.com/releases/news.aspx?id=1801>. Acesso em: 13 de Set. de 2016.

IDC; Smartphone vendor Market share, 2016. Disponível em: < http://www.idc.com/promo/smartphone-market-share/vendor> Acesso em: 08 de Mai. De 2017.

KRUG, S. Simplificando coisas que parecem complicadas. $1^{\circ}$ Edição. Altas Book, Rio de Janeiro, 2010.

MAFRA, S. Elaboração de chack list para desenvolvimento de projetos eficientes de cozinhas a partir de mapas mentais e escala likert. 1999. 188 f. Tese (Doutorado em Engenharia de Produção) - Departamento de produção e sistemas, Universidade federal de Santa Catarina, Santa Catarina. 1999.

MATTOS, T. Modelos Não Lineares e suas Aplicações. Trabalho de conclusão de curso de bacharelado em Estatística. Universidade Federal de Juiz de Fora, 2013.

MORAN, T. P. The command language grammars: a representation for the user interface of interactive computer systems. International Journal of ManMachine Studies, London, v. 15, n. 1, p. 3-50, July 1981.

MONTGOMERY, D.C.; RUNGER, G.C. Estatística aplicada e probabilidade para engenheiros. 5. ed. LTC. 2012

MUKAKA, M.M. Statistics Corner: A guide to appropriate uso of Correlation coefficient in medical research. Malawai Medical Journal. 2012.

NAGHETTINI, M.; PINTO, E. J. A. Hidrologia Estatística. 1a ed. Belo Horizonte: CPRM Companhia de Pesquisa de Recursos Minerais - Superintendência Regional de Belo Horizonte, 2007.

NIELSEN, J. Usability Engeneering. $1^{\circ}$ Ed. Academics Press, Inc. Londres, Inglaterra. 1993.

NIELSEN, J.; LORANGER, H. Usabilidade na web: Projetando websites com qualidade. Rio de Janeiro: Editora Campos - Elsevier, 2007.

SILVA, S.D. Júnior; COSTA, F. Mensuração e Escalas de Verificação: uma Análise Comparativa das Escalas de Likert e Phrase Completion. PMKT 24 de Jun. de 2014. ISSN:2317-0123

SMART MUNDO. Sistemas operacionais que existem hoje para Smartphones. Disponível em: < http://smartmundo.com/sistemas-operacionais-para-Smartphones/>. Acesso em: 16 de Dez. de 2016.

VERGARA, S. C. Projeto e relatórios de pesquisa em administração. São Paulo: Atlas Editora, 2003. 